SECTION 7. Mechanics and machine construction.
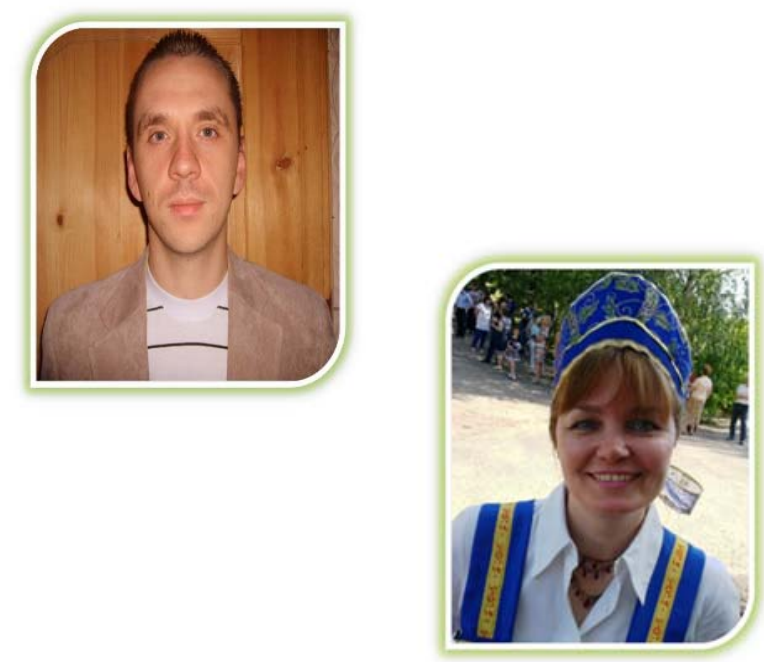

Denis Alexandrovich Chemezov Master of Engineering and Technology, Lecturer of Vladimir ChemicalMechanical College, Russia chemezov-da@yandex.ru

Anzhelika Vladimirovna Bayakina Lecturer of Vladimir Industrial College, Russia bajakina.anzhelika@yandex.ru

\title{
SIMULATION MODELING OF WATER FLOW IN THE VENTURI NOZZLE
}

Abstract: The article presents the results of simulation modeling: interactive plot and dependences of change of velocities of water flow in the Venturi nozzle.

Key words: water, the Venturi nozzle, mean velocity, cross section.

\section{УДК 66.011}

\section{ИМИТАЦИОННОЕ МОДЕЛИРОВАНИЕ ТЕЧЕНИЯ ВОДЫ В СОПЛЕ ВЕНТУРИ}

Аннотация: В статье представлены результаты имитационного моделирования: интерактивная эпюра и зависимости изменения скоростей течения воды в сопле Вентури.

Ключевые слова: вода, сопло Вентури, средняя скорость, сечение.

Сопло Вентури, наряду с нормальными соплами и диафрагмами, применяется для измерения переменного расхода жидкостей, газа и пара в трубопроводах [1, с. 115]. По сравнению с другими сужающими устройствами, сопло Вентури имеет ряд преимуществ: малые потери давления (5 ..20\% на выходе); высокая точность измерений и устойчивость к износу. Однако, громоздкая конструкция и установка устройства в трубопроводе с внутренним диаметром $(D)$ не менее 65 мм ограничивают широкое применение сопел Вентури на производстве [2, 8]. Конструктивно сопло Вентури представляет собой устройство, имеющее три профильных участка: сужающая радиусная входная часть длиной $0.304 d$ мм $(d-$ внутренний диаметр цилиндрической средней части сопла, определяется из соотношения $d=D / 1.8$ ); цилиндрическая средняя часть длиной $0.5 \ldots 0.7 d$ мм; диффузор (выходной конус с углом раскрытия $\psi=5 \ldots 30^{\circ}$ ) длиной не менее $3 d$ мм [3].

Известно, что изменение статического давления $P$ на различных участках сопла Вентури имеет следующий характер: резкое падение от номинального значения на длине сужающей радиусной входной части; постоянное на длине цилиндрической средней части; постепенное возрастание до установившегося значения, которое будет меньше номинального (потери энергии потока на преодоление сил трения) на длине диффузора [4].

Целью исследования являлось определение характера течения воды и последующий расчет средних скоростей в различных участках сопла Вентури, для оценки потери давления на выходе диффузора.

Исследование выполнялось в программной среде SolidWorks [5]. Для этого была построена трехмерная твердотельная модель сопла Вентури (рис.1). 


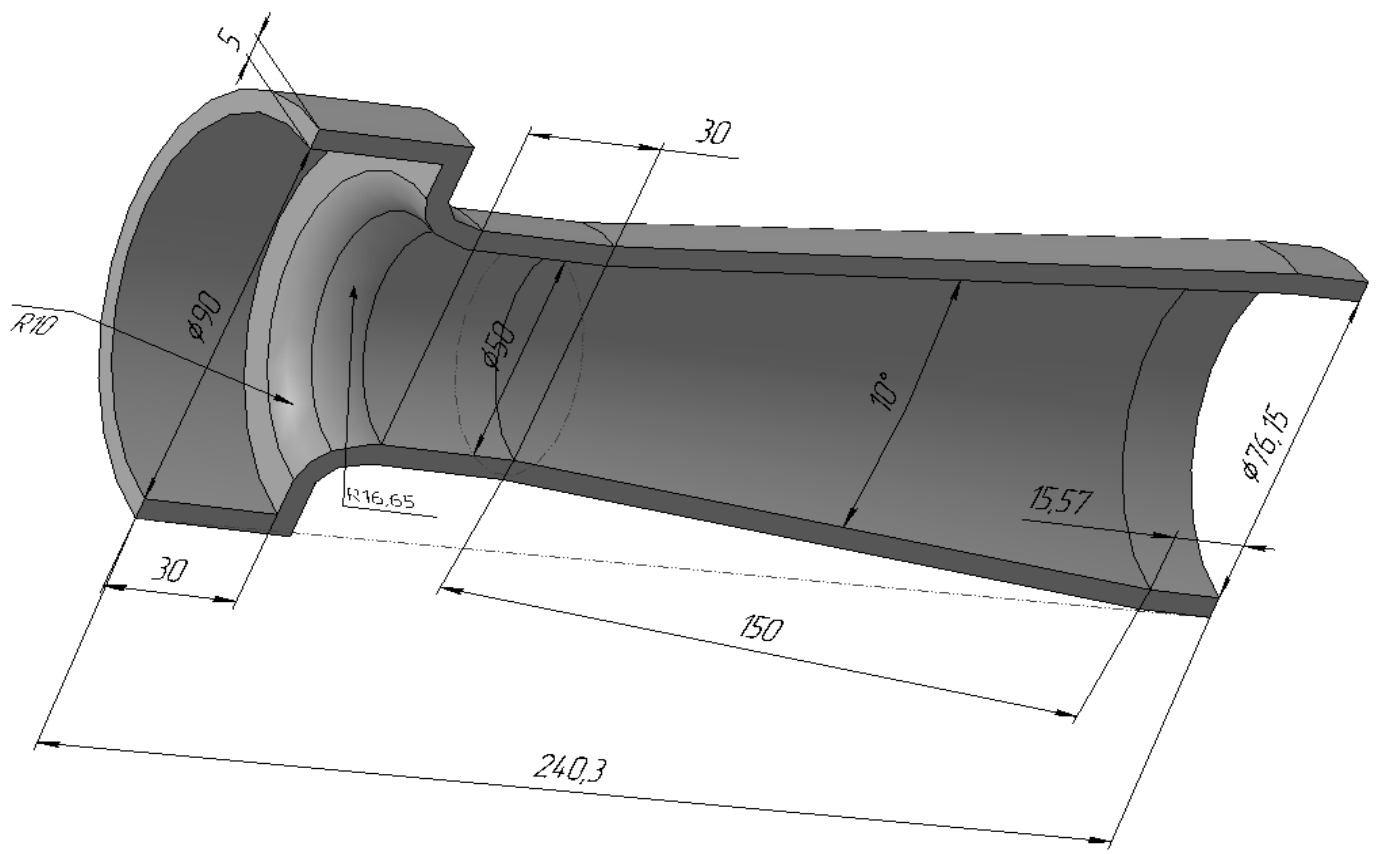

Рисунок 1 - Твердотельная модель сопла Вентури.

Для решения задачи гидродинамики в модуле FloXpress были приняты следующие граничные условия: наименьший проточный канал $d=50$ мм; жидкость - вода при температуре $22^{\circ} \mathrm{C}$; давление в впускном отверстии $P_{1}=200$ кПа; давление в выпускном отверстии $P_{2}=180$ кПа [6]. В качестве материала модели сопла Вентури принята нержавеющая сталь марки 12X18Н10T [7]. Результаты имитационного моделирования процесса течения воды через сопло Вентури представлены в виде эпюры результирующих скоростей (рис. 2).

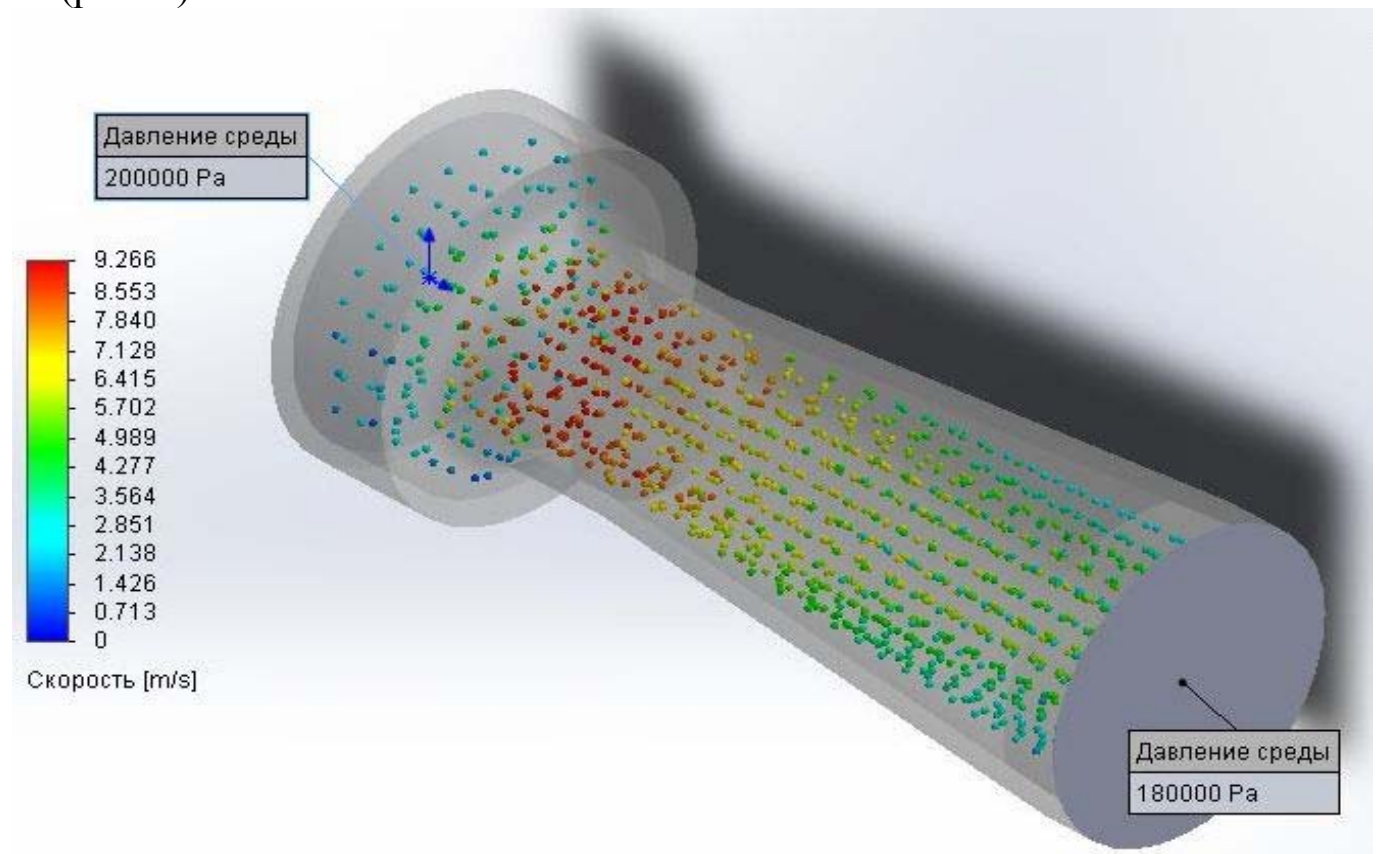

Рисунок 2 - Эпюра скоростей течения воды, действующих на различных участках сопла Вентури.

Слева от эпюры располагается шкала с гаммой цветов, напротив которой нанесены значения скорости течения (v) воды на всей длине сопла Вентури. Отмечено, что $v_{\max }=$ 
9,266 м/с (максимальная скорость течения) воды наблюдается в наименьшем сечении $d$, а минимальная $v_{\min }=0,713 \mathrm{~m} / \mathrm{c}$ во впускном отверстии (сечение трубопровода).

Рассмотрим динамику течения воды в диффузоре сопла. Разобьем длину диффузора $\left(l_{\text {диф. }}=150\right.$ мм) на 16 равных частей (рис. 3) и найдем среднюю скорость течения воды на площадях сечений. Расстояние между одноименными площадями сечений составило 9,375 MM.

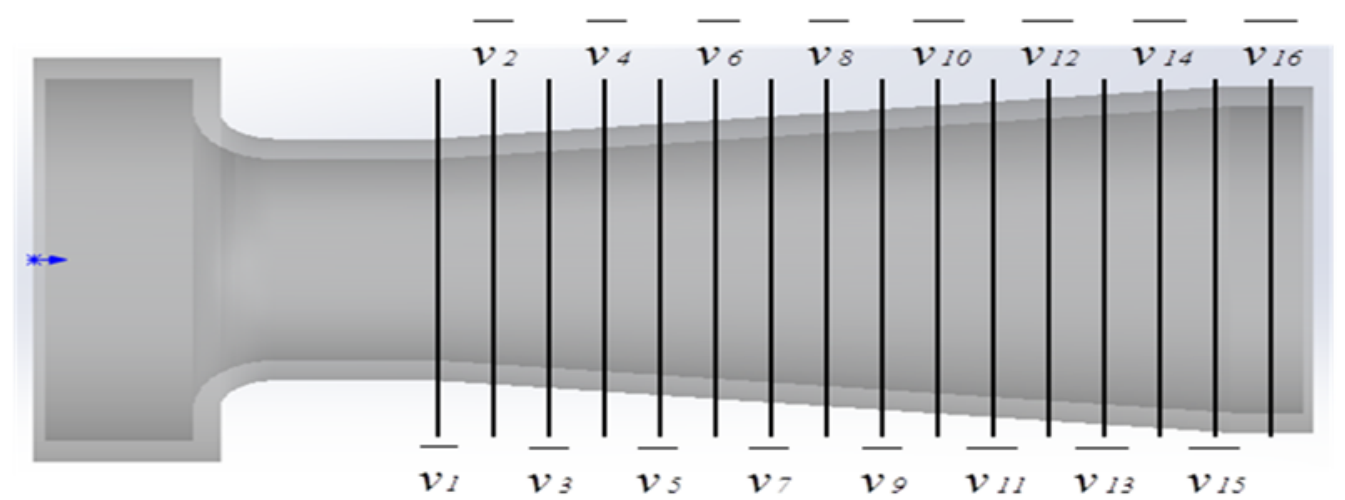

Рисунок 3 - Схема сечения длины диффузора для нахождения средних скоростей течения воды.

Средние скорости течения воды в рассматриваемых площадях сечений диффузора определяются по формуле (1)

$$
\overline{v_{\text {mean }}}=\frac{\mathrm{v}_{1} \mathrm{~m}_{1}+\mathrm{v}_{2} \mathrm{~m}_{2}+\mathrm{v}_{3} \mathrm{~m}_{3}+5+\mathrm{v}_{\mathrm{n}} \mathrm{m}_{\mathrm{n}}}{\mathrm{n}}=\frac{\sum_{\mathrm{i}=1}^{\mathrm{n}} \mathrm{v}_{\mathrm{i}} \mathrm{m}_{\mathrm{i}}}{\mathrm{n}},
$$

где $v_{1}, v_{2}, v_{3}, \ldots, v_{n}$ - скорости течения воды в сечениях диффузора (принимаются в диапазоне от 0,713 до $9,266 \mathrm{~m} / \mathrm{c}) ; m_{1}, m_{2}, m_{3}, \ldots, m_{n}$ - количество элементов разного цвета попавших в сечения диффузора (соответствуют скоростям течения воды), шт.; $n$ - сумма всех элементов попавших в сечения диффузора, шт.

Результаты расчета средних скоростей течения воды в сечениях диффузора представлены в таблице 1.

Таблица 1

Значения средних скоростей течения воды на площадях сечения диффузора сопла Вентури

\begin{tabular}{||c|c|c||}
\hline $\begin{array}{c}\text { Обозначение средней } \\
\text { скорости }\end{array}$ & Расчет параметра & $\begin{array}{c}\text { Значение параметра, } \\
\text { м/с }\end{array}$ \\
\hline \hline$\overline{v_{l}}$ & $\frac{7,128 \cdot 5+7,840 \cdot 5}{10}$ & 7,484 \\
\hline \hline$\overline{v_{2}}$ & $\frac{7,128 \cdot 6+7,840 \cdot 1+4,989 \cdot 3}{10}$ & 6,557 \\
\hline \hline$\overline{v_{3}}$ & $\frac{7,128 \cdot 6+7,840 \cdot 2+4,989 \cdot 2}{10}$ & 6,842 \\
\hline \hline$\overline{v_{4}}$ & $\frac{7,128 \cdot 7+4,989 \cdot 3}{10}$ & 6,486 \\
\hline \hline$\overline{v_{5}}$ & $\frac{7,128 \cdot 5+4,989 \cdot 5}{10}$ & 6,058 \\
\hline
\end{tabular}




\begin{tabular}{|c|c|c|}
\hline$\overline{v_{6}}$ & $\frac{6,415 \cdot 5+4,989 \cdot 5}{10}$ & 5,702 \\
\hline \multirow{2}{*}{$\overline{v_{7}}$} & $7,128 \cdot 5+7,840 \cdot 3+3,564 \cdot 2$ & \multirow{2}{*}{6,628} \\
\hline & 10 & \\
\hline \multirow{2}{*}{$\overline{v_{8}}$} & $3,564 \cdot 3+4,989 \cdot 7$ & \multirow{2}{*}{4,561} \\
\hline & 10 & \\
\hline \multirow{2}{*}{$\overline{v_{9}}$} & $3,564 \cdot 6+6,415 \cdot 2+4,989 \cdot 3$ & \multirow{2}{*}{4,471} \\
\hline & 11 & \\
\hline \multirow{2}{*}{$\overline{v_{10}}$} & $6,415 \cdot 2+5,702 \cdot 2+3,564 \cdot 7$ & \multirow{2}{*}{4,419} \\
\hline & 11 & \\
\hline \multirow{2}{*}{$\overline{v_{11}}$} & $2,851 \cdot 8+5,702 \cdot 3$ & \multirow{2}{*}{3,628} \\
\hline & 11 & \\
\hline \multirow{2}{*}{$\overline{v_{12}}$} & $2,851 \cdot 8+4,277 \cdot 3$ & \multirow{2}{*}{3,239} \\
\hline & 11 & \\
\hline \multirow{2}{*}{$\overline{v_{13}}$} & $2,851 \cdot 6+6,415 \cdot 2+4,989 \cdot 3$ & \multirow{2}{*}{3,822} \\
\hline & 11 & \\
\hline \multirow{2}{*}{$\overline{v_{14}}$} & $2,851 \cdot 5+4,277 \cdot 6$ & \multirow{2}{*}{3,628} \\
\hline & 11 & \\
\hline \multirow{2}{*}{$\overline{v_{15}}$} & $2,851 \cdot 6+4,989 \cdot 5$ & \multirow{2}{*}{3,822} \\
\hline & 11 & \\
\hline \multirow{2}{*}{$\overline{v_{16}}$} & $2,851 \cdot 5+4,277 \cdot 6$ & \multirow{2}{*}{4,010} \\
\hline & 11 & \\
\hline
\end{tabular}

Детальное описание рассматриваемого процесса демонстрирует график зависимостей скорости течения воды от длины диффузора сопла Вентури (рис. 4).

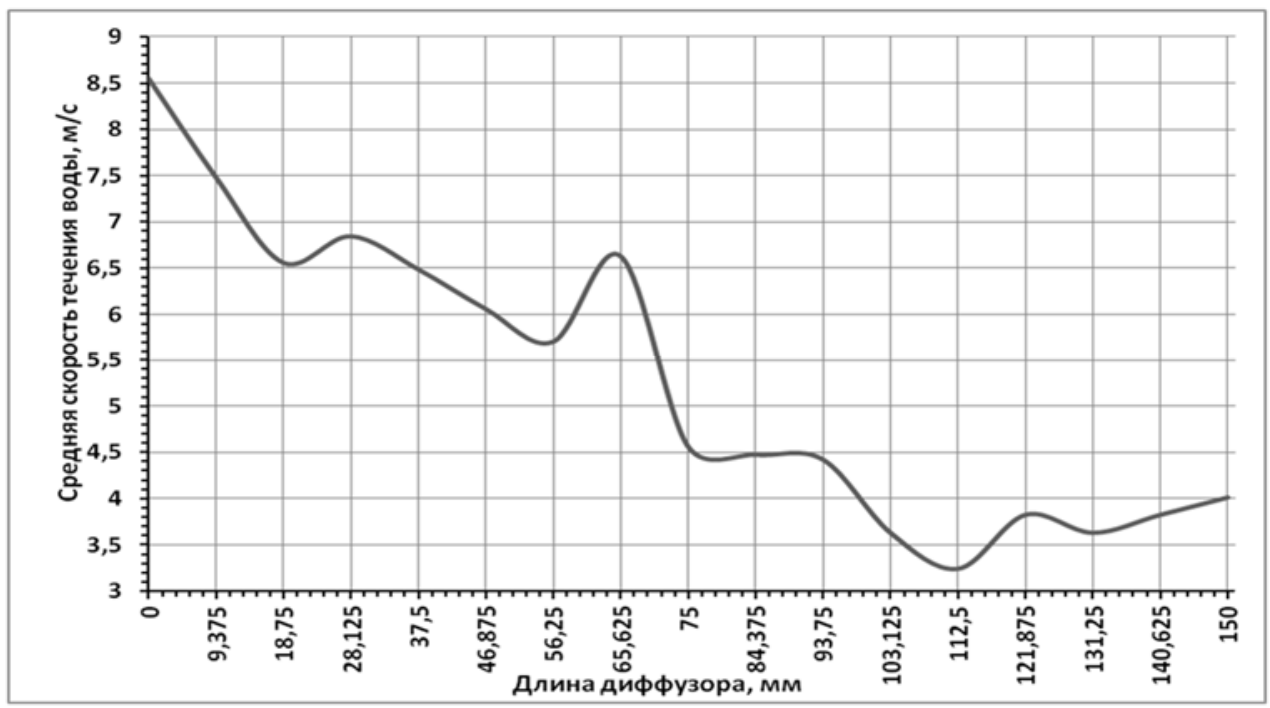

Рисунок 4 - Зависимость средней скорости течения воды от длины диффузора. 
Зависимость характеризуется нелинейными функциями: возрастающими и убывающими средними скоростями течения воды на всей длине диффузора. Это выражается тем, что потоки жидкости, проходящие через выходную расширяющуюся часть сопла, имеют различные скорости течения, распределенные по всему сечению - скорость течения верхних потоков снижается быстрей, чем нижних (турбулентное течение). Перепад давлений до профильной входной части сужающего устройства и после конической выходной части диффузора будет пропорционально зависить от неравномерности этих скоростей.

\section{References:}

1. Мелюшев Ю. К. Основы автоматизации химических производств / Ю. К. Мелюшев. М.: «Химия», 1973. -368 с.

2. Высокая точность сопел Вентури [Электронный ресурc] URL: http://www.bioaquaculture.ru/rotacionnyj-schetchik-s-pryamymi-lopastyami-6.html (дата доступа: 11.07.2014).

3. ГОСТ 8.586.3-2005. Измерение расхода и количества жидкостей и газов с помощью стандартных сужающих устройств. Часть 3. Сопла и сопла Вентури. Технические требования.

4. Измерение параметров жидкостей и газов [Электронный pecypc] URL: http://megapaskal.ru/newmen/768-normalnoe-soplo-venturi.html (дата доступа: 11.07.2014).

5. Уроки по SolidWorks [Электронный pecypc] URL: http://www.swlessonmpl.ru/index.php?start=48 (дата доступа: 11.07.2014).

6. Задание условий впускного отверстия потока [Электронный ресурс] URL: http://help.solidworks.com/2014/Russian/SolidWorks/floxpress/r_setting_flow_inlet_conditio ns.htm?id=58566e1558e144c0a4f9975d5ee95570\#Pg0 (дата доступа: 11.07.2014).

7. Трубка Вентури [Электронный ресурс] URL: http://ru.wikipedia.org/wiki/Tрубка_Вентури (дата доступа: 11.07.2014).

8. Сопла специального назначения [Электронный ресурc] URL: http://www.mattech.cz/ru/dyzy/specialni-dyzy (дата доступа: 11.07.2014). 